Health

Elsevier Editorial System(tm) for Public

Manuscript Draft

Manuscript Number: PUHE-D-19-00264R1

Title: Physical activity attitudes among adolescents in Bangladesh

Article Type: Original Research

Keywords: Physical activity; Exercise; Attitude; Adolescents; Bangladesh

Corresponding Author: Dr. Asaduzzaman Khan, PhD

Corresponding Author's Institution: The University of Queensland

First Author: Nicola Burton

Order of Authors: Nicola Burton; Mohammad Kadir; Asaduzzaman Khan

Abstract: Objectives: The purpose of this study was to examine physical activity (PA) attitudes among adolescents in Bangladesh, and their associations with socio-demographic, lifestyle and activity-related factors.

Study design: Cross sectional study.

Methods: A total of 781 students (52\% female; mean age 14.3 years, SD 1.1) from eight secondary schools in Dhaka, Bangladesh completed a written questionnaire. Exploratory factor analysis was used to derive positive and negative PA attitude measures. Generalized estimating equations was used to examine the associations.

Results: The most frequent positive attitudes were PA would 'get or keep me in shape' (81\%), and 'be fun' (73\%). Common negative attitudes were PA 'would make me hot and sweaty' (51\%), and 'is hard work' (42\%). Multivariable analysis showed that positive PA attitudes were more likely among adolescents who had physical education classes, adolescents involved in school sports, adolescents who ate fruit and vegetables daily, and adolescents who had breakfast regularly. Negative PA attitudes were more likely among girls, adolescents who slept $\leq 8$ hours/night, and adolescents who were overweight or obese, and less common among adolescents who ate fresh fruit and vegetables daily.

Conclusions: This study suggests that PA interventions for adolescents should highlight benefits for being in shape and having fun as key components of positive PA attitudes, and address negative attitudes of PA being hot and hard work. Specific strategies may be needed to address negative PA attitudes among girls, adolescents who are overweight or obese, and adolescents with insufficient sleep. 
26 August 2019

The Editor

Public Health

RE: PUHE-D-19-00264 Physical activity attitudes among adolescents in Bangladesh

Dear Editor,

Thank you very much for giving us the opportunity to revise and resubmit our manuscript for possible publication in your esteemed journal. We want to thank the reviewers for positive feedback on our manuscript and suggestions to improve the content.

Our point-by-point responses to the comments from the reviewer has been provided separately as a response note. Please let us know if you need any additional information or explanation.

We look forward to hearing about the status of our revised manuscript in due course.

Yours sincerely,

A/Prof Asaduzzaman Khan, $\mathrm{PhD}$

School of Health and Rehabilitation Sciences

The University of Queensland

Brisbane QLD 4072 Australia

T +61 733467456

F +61 733651877

Ea.khan2@uq.edu.au 


\section{Responses to Reviewers' Comments: PUHE-D-19-00264}

\section{Reviewer \#1}

This paper gives an interesting description of how attitudes to physical activity could be associated with socio-demographic, lifestyle and activity for adolescents in a lower-middle-income country. This is highly relevant in relation to overweight and obesity that is a public health concern also in this context. I do however believe that the paper would gain on some clarifications, mainly in the Methods.

Response: We appreciate the reviewer's positive and constructive comments. We have revised the manuscript thoroughly in response to the reviewer's comments.

In the Methods, first paragraph it would be helpful to the reader to give information about how the schools were selected, how many of the invited students participated, instead of referring to another paper.

Response: Following the comment, we have included a detail description of how the schools and students were selected in this study (see last paragraph, page 4).

Regarding the Statistical Analysis it is unclear in which context the PA attitudes were dichotomized and why, before performing the EFA and/or the GEE? How were the other variables coded in the analysis (BMI is described)?

Response: Sorry for the misunderstanding caused. PA attitudes were dichotomised only to obtain descriptive frequencies. All other analyses, including the multivariable modelling, were based on scores derived from the 5-point Likert scale responses of the attitude items. In addition, we have moved the first paragraph of 'Statistical Analysis' sub-section to its end (see last paragraph, page 8). Furthermore, we have added a description about coding of other variables used in this study (see last paragraph, page 6).

The authors describe how scoring of negative and positive attitudes were derived and transformed to normal distribution, was this used in the GEE?

Response: Yes, the transformed scores were used in GEEs.

I would suggest that the last sentence in the last paragraph in Statistical Analysis is moved to the first paragraph in Methods.

Response: Good suggestion - we have moved the sentence to the second paragraph in Methods.

The final model of GEE with only significant associations are included. Is it possible to give a description of the steps to the final model?

Response: Good point - we have added a description of the steps to the final models in the Statistical Analysis sub-section (see $1^{\text {st }}$ paragraph, page 8 ).

It may also be helpful for the reader with a table including descriptive data regarding the lifestyle variables. 
Response: Following the reviewer's comment, we have added a table (\#2) in the revised manuscript to describe the lifestyle variables. This information is also summarised in the results section (see $2^{\text {nd }}$ paragraph, page 9).

In the Results the authors state they have adjusted for other factors in the model, which factors and why?

Response: Sorry for the confusion caused. In the revised version, we have added a description about how the variables were selected for the final models (see first paragraph, page 8). Explanatory variables were selected based on statistical significance of their association (at $15 \%$ level of significance) with the outcomes of interest. Once the variables were included in the multivariable models, they were adjusted for each other in explaining their association with the outcomes.

\section{Reviewer \#2:}

The study is valuable as it explores country-specific research which the authors have established as a gap in the evidence. Prior to publication I have some concerns which I would like to see addressed.

Response: We appreciate the reviewer's positive and constructive comments, which have been addressed in the revised manuscript.

* Tables 1 and 2 are duplicated within the paper -why is this?

Response: Sorry for this - it was by mistake and has been corrected.

* The research highlights are not a good representation of the results reported -with no mention of gender or healthy eating -despite these being key themes in the abstract.

Response: We have revised the highlights to include results on healthy eating and gender.

There is also a lack of clarity in the abstract and the implications section - examine the following sentence: -

"with a specific attention to those who are female, overweight or obese, and those with poor sleep and unhealthy eating habits."

I would read this as suggesting that there are two groups who need attention: -

1. Females who are overweight or obese

2. Those with poor sleep and unhealthy eating habits.

However this is not my interpretation of the results sections. Firstly, they need to be clear that each of the characteristics above are separate, so it is a list: females, people who are overweight/obese, those with poor sleep, those with unhealthy eating habits. If statistical analysis has been carried out to give the 2 groups shown above, it is not adequately reported. Given the overlap of the confidence intervals for females compared to males, the data does not strongly support this conclusion anyway -certainly not sufficiently to ignore the needs of the males which is what the conclusion and abstract seem to suggest.

Response: The abstract, results, discussion and implications text has been revised to clarify that these are separate groups of adolescents. We have revised the implications text to reduce the emphasis on female adolescents (see $2^{\text {nd }}$ paragraph, page 13 ). 
We are not sure about the reviewer's comment on adequacy of reporting results. We have presented the odds ratio of being female (compared to male) to report negative PA attitudes as 1.20 with $95 \%$ confidence interval $1.02-1.38, p=0.012$, which clearly demonstrate that being female was significantly associated with reporting negative PA attitude. Hence, we believe that the results support the conclusion.

Secondly, although the survey asked about a wide range of eating habits, the only ones that showed a correlation with negative PA attitudes were breakfast and fruit eating. If multiple unhealthy eating habits have been examined the authors need to be specific about which ones they mean

Response: Good point. In response to the first reviewer's comments, we have added a table [\#2] with lifestyle variables including adolescents' dietary behaviours in the revised manuscript. Although fast food intake and consumption of dairy product were associated with positive PA attitudes at the univariate level (at $15 \%$ level of significance), they were not significant (at $5 \%$ level of significance) in the multivariable model [\#1]. This information has been added as a footnote of Table 3 . We have also revised the manuscript text to be more specific about which eating habits were associated with PA attitudes.

* The authors have chosen to use a five-point Likert scale with a "neutral" answer, but then presented the results as a dichotomous choice -agreed, or not agreed. This is probably due to this paper being written as a secondary analysis of data collected for another purpose, but I would suggest that the authors need to address this in their discussion and explore whether this threatens the validity of the conclusions drawn. If planning a survey for a dichotomous analysis, surely the questions would have forced a choice. What impact does this have on the results?

Response: As reported in the revised manuscript, dichotomisation of the attitude item responses (agree, not agree) was used only to provide descriptive results; all the other analyses including EFA, GEEs were based on the original 5-point Likert scale scores, and as such, the modelling results and the driven conclusion were unlikely to be affected by dichotomisation. This has been indicated in the discussion of the revised manuscript (see $2^{\text {nd }}$ paragraph, page 12).

* There is a lack of consistency in the manuscript about the approach to negative and positive attitude. On P4 Line 34, the authors state that negative attitudes are a stronger predictor of physical activity than positive attitudes. Granted, the paper referenced refers to pre-adolescents, but having included that information, the authors make no further mention of this difference. Their conclusion then focuses solely on positive attitudes with no explanation.

Response: The conclusions in the abstract and manuscript have been revised to refer to both positive and negative attitudes.

* Finally, I am concerned that a recommendation of the paper is that physical activity should be of low to moderate intensity (P11, Line 8) which is at odds with the very WHO guidelines which the paper purports to seek to support.

Response: This text has been deleted, and other more relevant information from the WHO recommendations about PA provided (see pages 12-13). 
Physical activity attitudes among adolescents in Bangladesh

N.W. Burton $\mathrm{PhD}^{1}$, M.A. Kadir $\mathrm{PhD}^{2}$, A. Khan $\mathrm{PhD}^{3^{*}}$

${ }^{1}$ School of Applied Psychology, Griffith University, Brisbane, Australia

${ }^{2}$ Social Marketing @ Griffith, Griffith University, Brisbane, Australia

${ }^{3}$ School of Health and Rehabilitation Sciences, The University of Queensland, Brisbane, Australia

Acknowledgements:

We thank the students and their parents who participated in the study, and the teachers and principals of the participating schools for their support of this project. Thanks to Parveen A Khanum, Shuhana Sultana, Nazneen A Khanum, Areebah S Khan and Shanchita R Khan for their assistance with data collection; Mahfuzul Kabir of Data Analysis and Technical Assistance (DATA) Bangladesh for his help with data entry, and Ms Rushdia Ahmed of icddr,b for providing comments on an earlier draft of the manuscript.

Declaration of interest:

The authors declare that they have no conflict of interest, real or perceived.

${ }^{*}$ Corresponding author:

Associate Professor Asaduzzaman Khan, $\mathrm{PhD}$

School of Health and Rehabilitation Sciences

The University of Queensland

Brisbane QLD 4072 Australia

T +61733467456

F +61733651877

E a.khan2@uq.edu.au 


\title{
Physical activity attitudes among adolescents in Bangladesh
}

\begin{abstract}
Objectives: The purpose of this study was to examine physical activity (PA) attitudes among adolescents in Bangladesh, and their whether such attitudes were-associations ed-with sociodemographic, lifestyle ander activity-related factors.
\end{abstract}

Study design: Cross sectional study.

Methods: A total of 781 students (52\% female; mean age 14.3 years, SD 1.1) from eight secondary schools in Dhaka, Bangladesh completed a self administered-written questionnaire which included 12 items about PA attitudes. Exploratory factor analysis was used to derive positive and negative PA attitude measures. Generalized estimating equations (GEE)-was used to examine the associations.

Results: The most frequently reperted positive attitudes were PA would 'get or keep me in shape' (81\%), and 'be fun' (73\%), and 'give me energy' (58\%). Commonly reported negative attitudes were PA 'would make me hot and sweaty' (51\%), and 'is hard work' (42\%), and 'requires serious commitment' (39\%). Multivariable analysis showed that positive PA attitudes were significantly-more likely among adolescents who had physical education classes, adolescents daily, and adolescents who had breakfast regularly. Negative PA attitudes were significantly more likely among females girls, adolescents those with insufficient who slept $\leq \underline{8}$ hours/nightsleep, and adolescents who were overweight or obese, and less common among adolescents those who ate fresh fruit and vegetables daily.

Conclusions: This study suggests that PA interventions for adolescents should highlight promote-benefits for being in shape and having fun as key components of positive PA attitudes, and address negative attitudes of PA being hot and hard work. aspects and benefits for being in shape and re-energising with a specific attention to. Specific strategies may be 
1

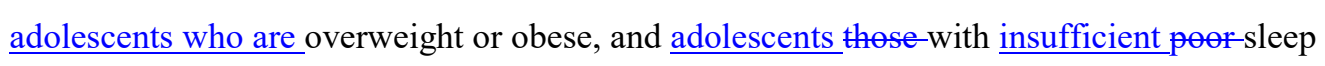
and unhealthy eating habits.

Keywords: Physical activity; Exercise; Attitude; Adolescents; Bangladesh 


\section{Introduction}

Among adolescents, physical activity (PA) has beneficial effects for self-concept, self-esteem, internalising and externalising problems, cognitive performance, academic achievement, self-reported health, weight, anxiety and depression ${ }^{1-3}$. PA, in particular

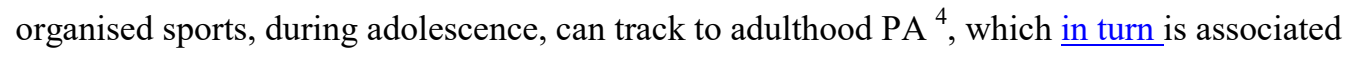
with a reduced risk of all-cause mortality and the primary and secondary prevention of chronic medicalconditions including cardiovascular diseases, type-2 diabetes, and several types of cancers ${ }^{5,6}$. However, a significant proportion of school-aged adolescents are not sufficiently active, with four out of five not meeting the World Health Organization (WHO) recommendations of $60 \mathrm{~min} /$ day of moderate-to-vigorous PA (MVPA) ${ }^{7}$.

Motivation is a key psychological correlate of PA participation among adolescents ${ }^{8}$, and is derived in part from attitudes which reflect positive or negative evaluations of engaging in the behaviour ${ }^{9}$. A positive attitude toward PA is positively associated with PA participation among adolescents ${ }^{10,11}$, and higher levels of PA after five and ten years, independent of baseline behaviour ${ }^{12}$. Research with pre-adolescents suggests that negative attitudes are a stronger (inverse) predictor of PA than positive attitudes ${ }^{13}$.

Attitudes towards PA can vary by socio-demographic and activity-related factors.

Less positive attitudes towards PA, including intensive exercise, sporting competitions and er sport, have been found among obese adolescents in Belgium and Germany ${ }^{14,15}$. R, and research in Tunisia suggests this may be moderated by that overweight and obesity were significantly associated with negative attitudes towards PA among those not meeting the recommended levels of PA, but no difference in perceptions by weight status among those who were meeting the PA recommendations ${ }^{16}$. An American study indicated that high school students who were male, students who were in a higher grade, and those who had with lower BMI had more positive PA attitudes than their counterparts ${ }^{17}$. PA attitudes may also 
be associated with other socio-demographic and lifestyle factors previously identified as correlates of adolescent PA, such as maternal education and family income ${ }^{18}$, healthy eating practice $^{19,20}$, and screen time ${ }^{21}$; however little work has been done to assess such relationships in adolescents in resource-poor settings.

Attitudes can also-be shaped by experiences and cultural influences ${ }^{9}$, so countryspecific research on PA attitudes is important due to between country variations in sociocultural, economic, and environmental conditions. Previous Earlier-research has indicated that people from Asia and women in Bangladesh have specific cultural beliefs and attitudes towards exercise ${ }^{22,23}$. We are not aware of any research on PA attitudes among adolescents in Bangladesh ${ }_{i}$, where, nationally, $59 \%$ of adolescents dide not meet the PA recommendations, according to the Global School based Health Survey ${ }^{24}$. The aims of this study were therefore, to assess positive and negative PA attitudes of adolescents from Dhaka eity -in Bangladesh, and the socio-demographic, lifestyle, and activity-related factors associated with PA attitudes.

\begin{abstract}
Methods
A total of 11 secondary schools in Dhaka, the capital city of Bangladesh, were invited to participate in the study and eight agreed. All students (aged 13-17 years) in grades 7-10 of these schools were then invited to do the survey. With permission from the headmasters/ principals of the schools and class teachers, the principal investigator (AK) attended classes and explained the rationale, purpose and requirements of the study. A written information sheet and consent form were distributed to students to be given to their parents/guardians for $\underline{\text { their review and consent for the adolescents and parents to participate. After obtaining the }}$ individual written informed consent from the students and their parents/guardians, students completed the survey in the classroom. The principal investigator (AK) and a class teacher
\end{abstract}


were present to monitor progress and to answer any questions or address any concerns the students had. Nonconsenting students engaged in alternate activities of their choice during $\underline{\text { this time. A section of the survey was given to students to take home and completed by one }}$ parent/guardian of the participating student to provide family-level data. The completed survey was returned by the student to the research team the following day. The survey was available in English and Bangla [the local language] and took approximately 45-50 minutes to complete. Data for this study were collected during the winter season (November 2012 to January 2013). Of the 1476 surveys distributed across the eight participating schools, 898 students responded. The study was approved by the Behavioural Social Sciences Ethics Review Committee at The University of Queensland, AustraliaData for this study were from a cross-sectional survey conducted among students of eight secondary schools purposively selected from Dhaka, the capital city of Bangladesh. More details about the study are available elsewhere. All students in grades 710 at the selected schools were invited to participate in the study. The research was approved by the Behavioural Social Sciences Ethics Review Committee.

\section{Measures}

Participants completed a self administered written questionnaire.Attitudes toward PA were measured by 12 items scored on a 5-point Likert scale with response options ranging from $1=$ strongly disagree to $5=$ strongly agree. The student questionnaire also included items to assess socio-demographic (gender, age, private or public school), physical health, life-style (sleep duration; frequency of consuming each of fast food, fresh fruit and vegetables, dairy products, sugary drinks, and breakfast) and activity-related (participation in physical education classes, involvement with team and non-team sports at school, screentime) factors. Parents of the participating students provided-Hhousehold/family level data provided by the parent/guardian of the participating students, includeding education, 
occupation, and family income, via a written questionnaire. Of the 898 students who participated in the survey, 781 (87\%) completed the PA attitude items and formed the analytical sample.

Participants' height and weight were measured by the research team members, and Centers for Disease Control and Prevention (CDC) growth charts were used to determine the corresponding body mass index (BMI) for-age and sex percentile ${ }^{25}$. Obesity was defined as a BMI at or above the 95th percentile for children of the same age and sex; overweight was a BMI between the $85^{\text {th }}$ and 95 th percentiles, healthy weight was a BMI less than the 85 th percentile but at or above the 5th percentile, and underweight was a BMI less than the 5th percentile. Categories of overweight and obese were combined to facilitate interpretation of comparisons.

Consumption of fruit and vegetable was assessed using two items (fresh fruit, salad or fresh vegetables) and responses dichotomised as at least once a day during the past week or less than once a day in the past week. Dairy intake (milk or milk products such as yoghurt or cheese) was assessed using one item and responses dichotomised as at least once a day during the past week or less than once a day in the past week. Fast food consumption (meals or snacks) was assessed using one item and responses dichotomised as at least once during the past week or none in the past week. Sugary soft drink intake was assessed using one item and responses categorised as 3 or more, $1-2$, or no cans, bottles or glasses in the past week. Beakfast frequency was assessed using one item and responses categorised as regular breakfast, which was defined as having breakfast on 5-7 days during the past week, and irregular breakfast which was having breakfast on 1-4 days in the past week. Sleep duration was assessed using one item and responses categorised as $<7$ hours, 7-8 hours and $>8$ hours in a usual night. 


\section{Statistical Analysis}

For each of the PA attitude items, responses were collapsed into a dichotomous grouping of 'agreed' ('agree' or 'strongly agree') and 'not agreed' ('neutral', 'disagree' or 'strongly disagree'). The proportion of adolescents who agreed with each of the positive and negative PA attitudes is presented in Figure 1.

To examine whether the $12 \mathrm{PA}$ attitude items could be summarized through unobserved constructs or factors, exploratory factor analysis (EFA) using principal components analysis extraction method and Varimax rotation was performed. Only eigenvalues over one were accepted for extraction of factors, and a cut-off of $>0.45$ was used for factor loadings. A high value (0.82) of the Kaiser-Meyer-Olkin measure of sampling adequacy and significance of the Bartlett test of sphericity $(p<0.001)$ suggested that the factor analysis was appropriate for the survey data. The factor analysis offered a two-factor solution explaining $53 \%$ of the variance. The first factor was labelled "positive attitudes", explained $29 \%$ of variance, and included six items: (i) help me cope with stress; (ii) be fun; (iii) help me make new friends; (iv) get or keep me in shape; (v) make me more attractive; and (vi) give me energy. The second factor was labelled "negative attitudes", explained $24 \%$ of the variance, and included six items: (i) take a lot of effort; (ii) require serious commitment; (iii) make me tired; (iv) take too much time; (v) make me hot and sweaty; and (vi) be hard work. Analyses indicated satisfactory reliability coefficients for both of the factors with Cronbach's alpha $=0.87$ and 0.83 , respectively, for positive and negative attitude items. Participant scores reflecting positive attitudes and negative attitudes were derived by taking the average of the

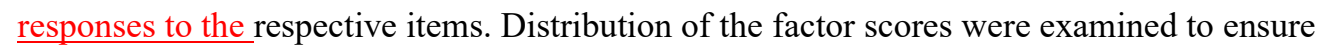
normality, and a square transformation was implemented on positive attitude scores to obtain a normal distribution. 
To examine whether positive and negative PA attitude_scores differed by any of the socio-demographic, lifestyle, or activity-related factors, we used Generalized Estimating Equations (GEE) which takes into account the non-independence of adolescents' attitudes toward PA nested within their schools. Initially, all potential explanatory variables were examined at the univariate level to assess their association with the outcomes of interest. A less conservative significance threshold of $15 \%$ was used at the univariate level to screen explanatory variables for subsequent multivariable analyses. For modelling the variables associated with positive PA attitudes, nine variables were associated with the outcome of interest at the univariate level, and four retained their significance (at 5\% level of $\underline{\text { significance) at the multivariable level (model 1). For modelling the variables associated with }}$ $\underline{\text { negative PA }}$ attitudes, seven variables were associated with the outcome of interest at the univariate level, and four retained their significance (at 5\% level of significance) at the multivariable level (model 2). Variables associated with positive and negative PA attitudes at the univariate level but not significant at the multivariable level are listed in the notes of Table 3. Residuals of the fitted models were examined to ensure normality of residuals and free from outliers. Only significant variables associations at from the final multivariable models are presented in the form of odds ratios (OR) and their 95\% confidence intervals (CI). All tests were performed at 5\% level of significance. Of the 898 students who participated in the survey, $781(87 \%)$ completed the attitude items and formed the analytical sample.

$\underline{\text { To describe the PA positive and negative attitudes among adolescents, item responses }}$ were collapsed into a dichotomous grouping of 'agreed' ('agree' or 'strongly agree') and 'not agreed' ('neutral', 'disagree' or 'strongly disagree'). The proportions of adolescents who agreed with each of the positive and negative PA attitudes are presented in Figure 1. 


\section{Results}

A summary of the chacteristics of the study participants is provided in Table 1 . The average age of the participants was $14.3(\mathrm{SD}=1.14)$ years, and half $(52 \%)$ were female. Parent level data indicated that $76 \%$ of the fathers and $58 \%$ of the mothers of the participants had tertiary level education, and $47 \%$ reported a monthly family income less than Taka $50,000$.

\section{Table 1 about here}

Lifestyle factors of the participating adolescents are presented in Table 2. Just less than a quarter $(23 \%)$ of adolescents reported having both fresh fruit and vegetables at least daily, and $41 \%$ consumed dairy products at least daily, during the past week. Consumption of carbonated sugary drinks was considerably high with a quarter of adolescents reporting having had at least three bottles/cans/glasses of sugary soft drinks during the past week. A third of the adolescents had fast food at least once during the past week, while over twothirds (70\%) had breakfast regularly (5-7 days/week).

\section{Table 2 about here}

As shown in Figure 1, the most frequently reported positive attitudes were that PA would 'get or keep me in shape' (81\%), 'be fun' (73\%), and 'give me energy' (58\%). The

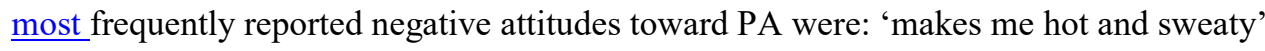
(51\%), 'hard work' (42\%), and 'requires serious commitment' (39\%).

\section{Figure 1 about here}

A summary of the multivariable GEE modelling results is provided in Table $\underline{3} z$. After adjusting for other factors, there were higher odds of reporting positive PA attitudes among adolescents who had physical education classes at school, adolescents sports (team or non-team) at school, adolescents who ate fresh fruit and vegetables at least 
dailyevery day, and adolescents who had breakfast regularly (5-7 days/week). Negative PA attitudes were more likely among female adolescents who were female, adoelscents who $\underline{\text { were }}$-r overweight/obese, and adolescents who had insufficient sleep (slept $\leq 8$ hours/night). Negative PA attitudes were less likely among adolescents who ate fresh fruit and vegetables at least dailyevery-day in the past week.

\section{Table 32 about here}

\section{Discussion}

This study assessed positive and negative PA attitudes of school-based adolescents from Dhaka city in Bangladesh, and the associated socio-demographic, lifestyle, and activityrelated factors. The most common positive PA attitude was that PA would 'get or keep me shape'. This is consistent with other research on adolescents' expectations of PA ${ }^{26-28}$, and research indicating body shape attitudes as a predictor of adolescent PA participation ${ }^{29,} 30$. The second leading positive attitude was that PA would 'be fun'. Fun has previously been identified as an important factor for adolescent PA ${ }^{30-33}$, and is associated with diversified activities, challenge but not competition, positive practice experiences, presence of friends, no parental pressure, perceived competence, and autonomy ${ }^{32}$.

Positive PA attitudes were more likely among adolescents who had physical education classes and adolescents involved in school sports, which supports other studies ${ }^{10,11}$. It may be that these experiences promote positive PA attitudes by providing time with friends, and social support and encouragement from school-based leaders. Other research has identified that school-based $\underline{\mathrm{PA}}$ opportunities and motivating teachers are a-key enablers of PA among adolescents in India ${ }^{28}$. Our study also found that positive attitudes towards PA were more common among adolescents who ate fruit and vegetables at least daily; and adolescents who ate breakfast regularly (5-7 days/week). This could reflect the 
results of other research indicating that active adolescents relate both exercise and nutrition with the definition of being healthy ${ }^{34}$.

Other research indicates that nNegative attitudes may be a stronger (inverse) predictor of PA than positive attitudes ${ }^{13}$. In the current study, common negative attitudes among adoelscents were PA 'would make me hot and sweaty' and 'is hard work'. As a tropical country, Bangladesh has a hot and humid climate. Poor or extreme weather has previously been identified as a barrier to $\mathrm{PA}^{35}$, and people living in areas with moist tropical conditions have lower PA levels than those with dry moderate conditions ${ }^{36}$. Perceptions of PA as hard work can reflect low activity efficacy, low perceived competence, competing time demands or lack of support and encouragement, which are all barriers to adolescent PA ${ }^{32}$.

Negative PA attitudes were more likely among female adolescents-who were female,

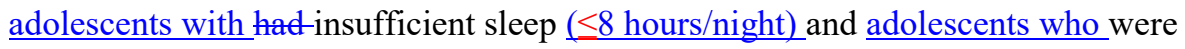
overweight or obese; and less common among adolescents those-who ate fruit and vegetables at least daily. For some adolescent girls, PA and sport is inconsistent with feminine stereotypes, and therefore risks negative judgements ${ }^{32}$. This may be particularly salient in countries like Bangladesh where there are strong gender norms and roles. Other research in India highlighted that sociocultural expectations can be strong deterrents to PA among adolescent girls, and relate to the appropriateness of types of PA, clothes worn for PA and who PA is done with ${ }^{28}$. Geting in ${ }^{28}=$ Adolescent girls are also more likely than boys to report low activity-efficacy and high perceived exertion for PA ${ }^{28,37}$. Adolescent girls may also be concerned about safety or and neglecting studies and homework as a result of PA participation ${ }^{28}$. Getting hot and sweaty was the most common negative PA attitude in our study, and is a common negative perception of PA among adolescent girls in general 27,28 . Adolescents with insufficient sleep 
can experience fatigue, which is a barrier to PA ${ }^{38,39}$ and likely to contribute to negative PA attitudes of PA being hard workreflecting perceptions of effort and demand. Lack of sleep and being tired has previously been identified as a barrier to PA among adolescents girls in India $^{28}$. The positive association in our study between overweight/obesity and negative PA attitudes complements previous research demonstrating less positive attitudes towards PA among obese adolescents ${ }^{14,15}$. Similarly, the inverse association in our study between at least daily fruit and vegetable consumption healthy eating behaviours and negative PA attitudes reflects previously discussed research among young adolescents linking exercise and nutrition with the definition of being healthy ${ }^{34}$.

Data for this study were from a large urban sample with a good response rate. The study included both positive and negative PA attitudes, and explored a wide range of sociodemographic, lifestyle and activity-related factors as potentially associated with PA attitudes. Although the individual PA attitude item responses were dichotomised (agree, not agree) to obtain descriptive frequencies, the statistical analyses including EFA, GEEs used generated summative scores based on the original 5-point Likert scale responses. Participants were volunteers recruited from a limited number of schools in a metropolitan area, which may have can result in created selection bias. Results, therefore, may not be generalisable to all adolescents, in particular those in regional areas. Positive and negative PA attitudes were assessed using a limited number of items, and different results may have been obtained if different attitude items were assessed. Self-report of PA attitudes may be vulnerable to social desirability bias.

\section{Implications and Conclusions}

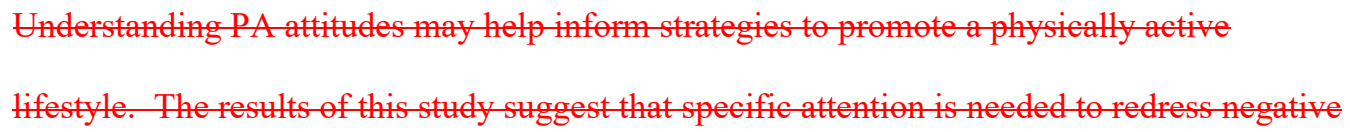


PA attitudes among Bangladeshi adolescents who are female, overweight or obese, and those with insufficient sleep and unhealthy eating habits. Multi-component interventions could therefore, simultaneously address healthy $\mathrm{PA}$, eating and sleep practices. PA interventions should promote the fun aspects and benefits for being in shape and re-energising. School based opportunities via physical education classes and sports may improve PA attitudes. Given the common negative attitudes of PA being 'hard work' and 'hot and sweaty', PA epportunities should cater for adolescents with weight concerns and low efficacy, be of low to moderate intensity, and be done in cool or climate controlled contexts. Among Bangladeshi adolescent girls, less "masculine" types of PA may be appealing, and the "feminine" benefits of $\mathrm{PA}$ could be highlighted. A physically active lifestyle among adolescents could track into adulthood and provide significant benefits for current and future health and wellbeing.

An understanding of positive and negative PA attitudes can inform PA interventions. The $\underline{\text { results of this study suggest that PA interventions for Bangladeshi adolescents could highlight }}$ benefits of being in shape and having fun, which contribute to positive PA attitudes. School based-opportunities via physical education classes and sports may improve positive PA attitudes. Some specific types of PA (e.g., water based activities) and PA conducted in $\underline{\text { climate-controlled environments (e.g., air-conditioned venues) may counter negative attitudes }}$ of PA being hot and sweaty. To address negative attitudes of PA being hard work, PA opportunities could cater for adolescents with low efficacy and/or capability. This could include activities that provide mastery experiences and are low impact or moderate intensity, as well as activities that can be integrated into the daily schedule such as active commuting. It could also be advantageous to highlight the value of low doses of PA. As identified in the World Health Organization recommendations for children aged 5-17 years, doing some PA, 
even if below the recommended levels, offers more benefits than doing none at all, and it is appropriate to start with small amounts of PA and gradually increase duration, frequency and $\underline{\text { intensity over time }}^{40}$. As negative PA attitudes were more common among adolescents with insufficient sleep and adolescents who did not eat fruit and vegetables daily, there could be a role for multi-component interventions that simultaneously address these three health-related behaviours. Specific strategies may also be needed to engage female adolescents who were more likely to have negative PA attitudes than male adolescents. Highlighting the "feminine" benefits of PA and promoting less "masculine" types of PA could potentially be appealing to the Bangladeshi girls. Future research could explore "feminine" stereotypes of exercise and activity types and contexts perceived as gender appropriate in this culture. Successful implementation of such PA interventions among adolescents could provide significant benefits for current and future health and wellbeing. 
1

2

\section{Funding}

This research did not receive any specific grant from funding agencies in the public, commercial, or not-for-profit sectors.

\section{Conflict of interest}

The authors declare that they have no conflict of interest, real or perceived. 


\section{References}

1. Spruit A, Assink M, van Vugt E, van der Put C, Stams G. The effects of physical activity interventions on psychosocial outcomes in adolescents: A meta-analytic review. Clin Psychol Rev. 2016; 45:56-71.

2. Granger E, Di Nardo F, Harrison A, Patterson L, Holmes R, Verma A. A systematic review of the relationship of physical activity and health status in adolescents. Euro $\mathrm{J}$ Public Health. 2017; 27:100-6.

3. Biddle S, Asare M. Physical activity and mental health in children and adolescents: a review of reviews. Br J Sports Med. 2011; 45:886-95.

4. Evans JMM, Sheila CM, Kirk A, Crombie IK. Tracking of physical activity behaviours during childhood, adolescence and young adulthood: a systematic review. J Epidemiol Community Health. 2009; 63:9.

5. Milton K, Macniven R, Bauman A. Review of the epidemiological evidence for physical activity and health from low-and middle-income countries. Glob Public Health. 2014; 9:369-81.

6. Warburton D, Bredin S. Health benefits of physical activity: a systematic review of current systematic reviews. Curr Opin Cardiol. 2017; 32:541-56.

7. Rhodes RE, Janssen I, Bredin SS, Warburton DE, Bauman A. Physical activity: Health impact, prevalence, correlates and interventions. Psychol Health. 2017:1-34.

8. Biddle S, Atkin AJ, Cavill N, Foster C. Correlates of physical activity in youth: a review of quantitative systematic reviews. Int Rev Sport Exercise Psychol. 2011; 4:25-49.

9. Ajzen I, Fishbein M. Understanding attitudes and predicting social behavior. Englewood Cliffs, NJ: Prentice Hall; 1980.

10. Van der Horst K, Paw M, Twisk JW, Van Mechelen W. A brief review on correlates of physical activity and sedentariness in youth. Med Sci Sports Exercise. 2007; 39:1241-50.

11. Lowry R, Lee S, Fulton J, Z D, Kann L. Obesity and other correlates of physical activity and sedentary behaviors among US high school students. J Obes. 2013; 2013:276318.

12. Graham D, Sirard J, Neumark-Sztainer D. Adolescents' attitudes toward sports, exercise and fitness predict physical activity 5 and 10 years later. Prev Med. 2011; 52:130-32.

13. Nelson T, Benson E, Jensen C. Negative attitudes toward physical activity: measurement and role in predicting physical activity levels among preadolescents. $\mathrm{J}$ Pediatr Psychol. 2010; 35:89-98.

14. Kopczynski S, Chen-Stute A, Kellmann M. Attitudes towards physical Activity and exercise participation-a comparison of healthy-Weight and obese Adolescents. Dtsch Z Sportmed. 2014; 65:139-43.

15. Deforche BI, De Bourdeaudhuij IM, Tanghe AP. Attitude toward physical activity in normal-weight, overweight and obese adolescents. J Adolesc Health. 2006; 38:56068.

16. Maatoug J, Harrabi I, Sahli J, Ezzi O, Chebil D, Gaddour M, et al. Attitudes toward physical activity according to weight status among schoolchildren in Sousse, Tunisia Health Serv Res Manag Epidemiol. 2015:doi.org/10.1177/2333392815601841.

17. Chen Y, Hwang J, Jung Y, Beddoes Z, Castelli D. Investigating attitudes toward physical activity among adolescents. Res Quart Exercise Sport. 2016; 87:A27. 
18. Gordon-Larsen P, McMurray R, Popkin B. Determinants of adolescent physical activity and inactivity patterns. Pediatrics. 2000; 105:e83.

19. Pearson N, Atkin A, Biddle S, Gorel T, Edwardson T. Patterns of adolescent physical activity and dietary behaviours. Int J Behav Nutri Phy Act. 2009; 6:45.

20. Corder K, van Sluijs E, Ridgway C, Steele R, Prynne C, Stephen A, et al. Breakfast consumption and physical activity in adolescents: daily associations and hourly patterns. Am J Clin Nutr. 2014; 99:361-68.

21. Kremer P, Elshaug C, Leslie E, Toumborous J, Patton G, Williams J. Physical activity, leisure-time screen use and depression among children and young adolescents. J Sci Med Sport. 2014; 17:183-87.

22. Patel N, Ferrer H, Tyrer F, Wray P, Farooqi A, Davies M, et al. Barriers and facilitators to healthy lifestyle changes in minority ethnic populations in the UK: a narrative review. J Racial Ethn Health Disparities. 2017; 4:1107-19.

23. Khanam S, Costarelli V. Attitudes towards health and exercise of overweight women. J R Soc Promot Health. 2008; 128:26-30.

24. World Health Organization. Global School-based Student Health Survey: Bangladesh 2014 Fact Sheet. 2014 [cited 2017 February 9]; Available from: http://www.who.int/chp/gshs/2014-Bangladesh-fact-sheet.pdf?ua=1.

25. Ogden C, Flegal K. Changes in terminology for childhood overweight and obesity. Natl Health Stat Rep. 2010; 25:1-5.

26. Gavin J, Mcbrearty M, Harvey M. Involvement in physical activity: adolescents' perceptions of outcomes. SAGE Open. 2013; 3:1-10.

27. Grieser M, Vu M, Bedimo-Rung A, Neumark-Sztainer D, Moody J, Rohm Young D, et al. Physical activity attitudes, preferences, and practices in African American, Hispanic, and Caucasian girls. Health Educ Behav. 2006; 33:40-51.

28. Satija A, Khandpur N, Satija S, Mathur Gaiha S, Prabhakaran D, Reddy K, et al. Physical activity among adolescents in India: a qualitative study of barriers and enablers. Health Educ Behav. 2018; 45:926-34.

29. Kahn J, Huang B, Gillman M, Field A, Austin S, Colditz G, et al. Patterns and determinants of physical activity in U.S. adolescents. J Adoelsc Health. 2008; 42:36977.

30. Butt J, Weinberg R, Breckon J, Claytor R. Adolescent physical activity participation and motivational determinants across gender, age, and race. J Phys Act Health. 2011; 8:1074-83.

31. Weiss M, Williams L. The why of youth sport invovlement: a development perspective on motivational processes. In: Weiss M, editor. Developmental sport an exercise physiology: A lifespan perspective. Morgantown, WV: Fitness information Technology; 2004.

32. Martins J, Marques A, Sarmento H, Carreiro da Costa F. Adolescents' perspectives on the barriers and facilitators of physical activity: a systematic review of qualitative studies. Health Educ Res. 2015; 30:742-55.

33. James M, Todd C, Scott S, Stratton G, McCoubrey S, Christian D, et al. Teenage recommendations to improve physical activity for their age group: a qualitative study. BMC Public Health. 2018; 18:372.

34. Belton S, O’Brien W, Meegan S, Woods C, Issartel J. Youth-Physical Activity Towards Health: evidence and background to the development of the Y-PATH physical activity intervention for adolescents. BMC Public Health. 2014; 14:122.

35. Tucker P, Gilliland J. The effect of season and weather on physical activity: A systematic review. Public Health. 2007; 121:909-22. 
36. Merrill RM, Shields EC, White GL, Druce D. Climate conditions and physical activity in the United States. Am J Health Behav. 2005; 29:371-81.

37. Robbins L, Pender N, Ronis D, Kazanis A, Pis M. Physical activity, self-efficacy, and perceived exertion among adolescents. Res Nurs Health. 2004; 27:435-46.

38. Kimm S, Glynn N, Mcmahon R, Voorhees C, Weissmann R, Daniels S. Selfperceived barriers to activity participation among sedentary adolescent girls. Med Sci Sports Exercise. 2006; 38:534-40.

39. Bragg M, Tucker C, Kaye L, Desmond F. Motivators of and barriers to engaging in physical activity: perspectives of low-income culturally diverse adolescents and adults. Am J Health Educ. 2009; 40:146-54.

40. World Health Organization. Recommendations levels of physical activity for children aged 5-17 years. [cited 201922 August]; Available from:

https:/www.who.int/dietphysicalactivity/factsheet young people/en/. 
Table 1 - Demographics of participating adolescents in Dhaka city, Bangladesh $(n=781)$

\begin{tabular}{|c|c|c|}
\hline Characteristics & $\mathbf{n}^{\mathrm{a}}$ & $\%$ \\
\hline \multicolumn{3}{|l|}{ Gender } \\
\hline GirlsFemale & 404 & 51.7 \\
\hline BoysMale & 377 & 48.3 \\
\hline \multicolumn{3}{|l|}{ Age in years } \\
\hline 13 & 192 & 24.6 \\
\hline 14 & 244 & 31.2 \\
\hline 15 & 228 & 29.2 \\
\hline 16 & 117 & 15.0 \\
\hline \multicolumn{3}{|l|}{ Mother's education } \\
\hline Up-to primary or equivalent & 67 & 8.8 \\
\hline Secondary or equivalent & 107 & 13.9 \\
\hline Higher secondary or equivalent & 147 & 19.2 \\
\hline Tertiary & 445 & 58.1 \\
\hline \multicolumn{3}{|l|}{ Mother's occupation ${ }^{\mathrm{b}}$} \\
\hline Employed & 174 & 25.9 \\
\hline Not employed & 497 & 74.1 \\
\hline \multicolumn{3}{|l|}{ Father's education } \\
\hline Up-to primary or equivalent & 47 & 6.1 \\
\hline Secondary or equivalent & 55 & 7.2 \\
\hline Higher secondary or equivalent & 83 & 10.9 \\
\hline Tertiary & 579 & 75.8 \\
\hline \multicolumn{3}{|l|}{ Father's occupation } \\
\hline Public service & 172 & 25.2 \\
\hline Professional & 98 & 14.3 \\
\hline Working on private organization & 219 & 32.1 \\
\hline Business-trade & 194 & 28.4 \\
\hline \multicolumn{3}{|l|}{ Family income $(\mathrm{BD} \text { Taka })^{\mathrm{c}}$ per month } \\
\hline$<30000$ & 167 & 22.8 \\
\hline $30000-<50000$ & 180 & 24.5 \\
\hline $50000-<100000$ & 216 & 29.4 \\
\hline 100000 or more & 171 & 23.3 \\
\hline
\end{tabular}



Table 2 - Lifestyle factors of participating adolescents in Dhaka city, Bangladesh ( $\mathrm{n}=781)$

\begin{tabular}{lll}
\hline Characteristics & $\mathbf{n}^{\mathrm{a}}$ & $\mathbf{\%}$ \\
\hline Fresh fruit-vegetable intake during the past week & & \\
$\quad$ Less than daily & 600 & 77.3 \\
$\quad$ At least daily & 176 & 22.7 \\
Consumption of dairy products during the past week & & \\
$\quad$ Less than daily & 462 & 59.4 \\
$\quad$ At least daily & 316 & 40.6 \\
Fast-food consumption during the past week & & \\
$\quad$ None & 515 & 67.4 \\
$\quad$ At least once & 249 & 32.6 \\
Have breakfast during the past week & & \\
$\quad$ Irregular (1-4 days) & 236 & 30.4 \\
$\quad$ Regular (5-7 days) & 541 & 69.6 \\
Sugary soft drink intake during the past week & & \\
(cans/bottles/glasses) & & \\
$\quad$ None & 267 & 34.4 \\
1-2 & 314 & 40.5 \\
$\quad 3$ or more & 195 & 25.1 \\
Average sleep duration in a usual night & & \\
$\quad<7$ hours & 143 & 22.0 \\
$\quad 7-8$ hours & 231 & 35.5 \\
$\quad>8$ hours & 277 & 42.5 \\
\hline a Total for each variable may not be equal to n=781 due to missing values. & &
\end{tabular}

${ }^{\mathrm{a}}$ Total for each variable may not be equal to $\mathrm{n}=781$ due to missing values. 
Table $\underline{3} z$ - Factors significantly associated with adolescents' positive and negative attitudes towards physical activity.

\begin{tabular}{lccc}
\hline Characteristics & $\begin{array}{c}\text { Odds } \\
\text { Ratio }\end{array}$ & $\begin{array}{c}95 \% \text { Confidence } \\
\text { Interval }\end{array}$ & $p$-value \\
\hline Model $1^{a}$ & \multicolumn{3}{c}{ Positive attitudes } \\
\hline
\end{tabular}

Physical education classes at school $(\mathrm{Ref}=\mathrm{No})$

$\begin{array}{lllll}\text { Yes } & 2.70 & 1.22 & 5.98 & 0.015\end{array}$

Sport involvement at school $(\operatorname{Ref}=\mathrm{No})$

Yes

Fresh fruit and vegetables at least daily-everyday $(\mathrm{Ref}=\mathrm{No})$

Yes

$10.18<0.001$

3.88

1.86

$8.08<0.001$

Regular breakfast (5-7days/week) (Ref=No)

\begin{tabular}{|lcccc|} 
Yes & 4.23 & 1.78 & 10.03 & 0.001 \\
\hline $\begin{array}{l}\text { Model } 2^{b} \\
\text { Gender (Ref=Male) } \\
\text { Female }\end{array}$ & 1.20 & 1.04 & 1.38 & 0.012
\end{tabular}

Weight status $(\mathrm{Ref}=\mathrm{Normal})$

Underweight

$\begin{array}{llll}1.15 & 0.90 & 1.46 & 0.27\end{array}$

Overweight or obese

$\begin{array}{llll}1.42 & 1.23 & 1.63 & <0.001\end{array}$

Average sleep duration in a usual nightduration

(Ref: $=\geq 8 \neq$ hours/day)

7-8 hours/day

1.41

1.21

$1.66<0.001$

$<7$ hourstday

1.38

1.19

$1.61<0.001$

Fresh fruit and vegetable at least dailyeveryday $($ Ref $=$ No)

Yes

0.80

0.66

0.98

0.029

${ }^{\mathrm{a}}$ Factors associated with positive PA attitudes at univariate level but not significant at the multivariable level include weight status, sleep duration, consumption of fast food and dairy products, and mother's education.

${ }^{\mathbf{b}}$ Factors associated with negative PA attitudes at univariate level but not significant at the multivariable level include school type (public vs private), mother's occupation and father's education. 


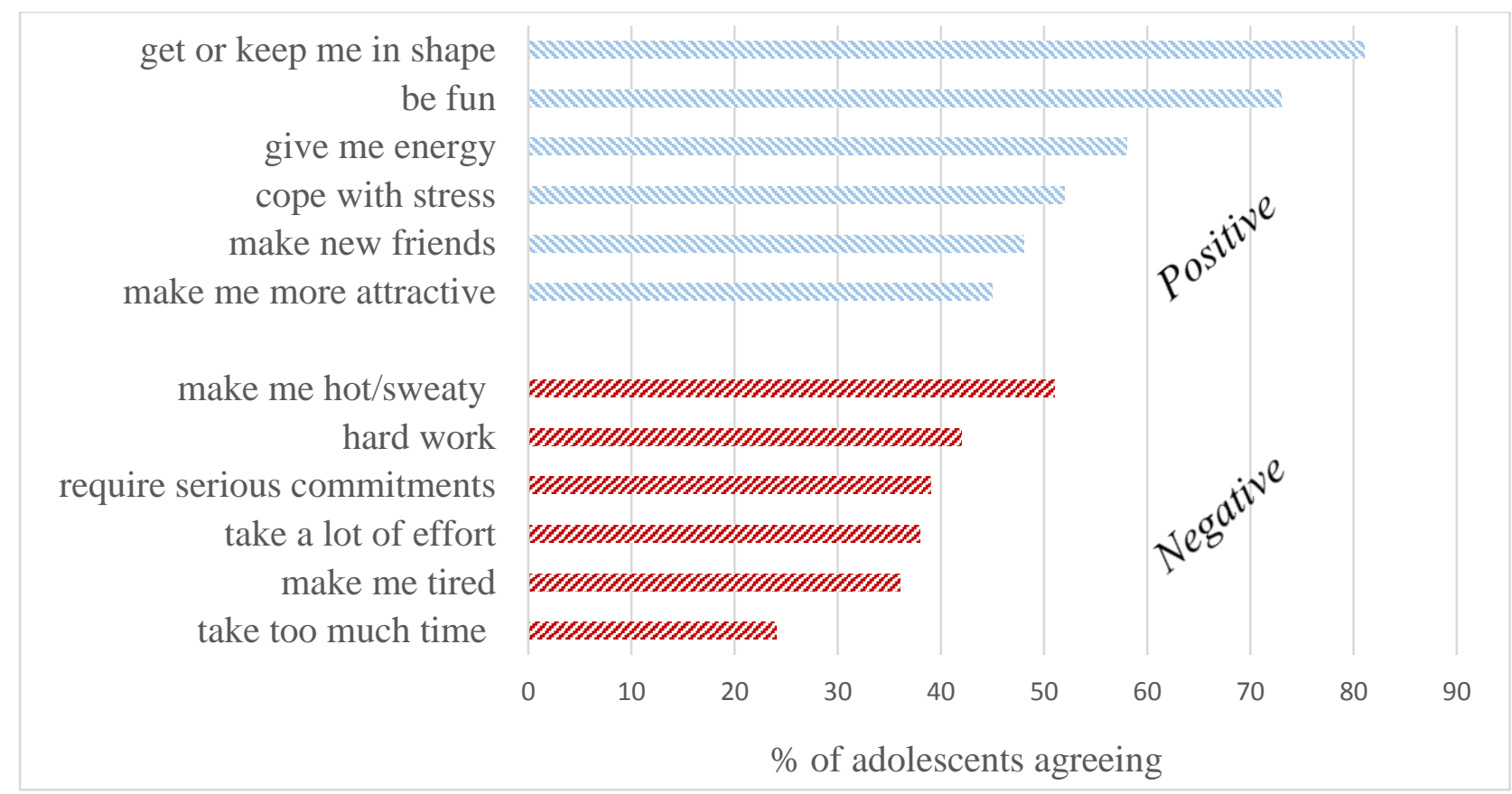

Figure 1. Proportion of adolescents agreeing with positive and negative attitude items toward physical activity. 


\section{Research highlights}

- Attending physical education class and school sports is linked to positive PA attitude.

- Insufficient sleep, and being overweight/obesity are linked to negative PA attitude.

- PA intervention should focus on promoting healthy eating, body image and energy.

- Common positive attitudes are that PA helps being in shape and is fun.

- Common negative attitudes are that PA is hot, sweaty and hard work.

- Physical education class and school sports are associated with positive PA attitudes.

- Adolescents who eat fruit, vegetables and breakfast daily have better PA attitudes.

- Girls and adolescents with insufficient sleep have more negative PA attitudes. 